\title{
ESTIMATE OF CORONAL MAGNETIC FIELD STRENGTH USING PLASMOID ACCELERATION MEASUREMENT
}

\author{
Minhwan JAnG ${ }^{1}$, G. S. ChoE ${ }^{2}$, K.-S. LeE ${ }^{1}$, Y.-J. Moon ${ }^{2}$, And KaP-Sung Kim ${ }^{2}$ \\ ${ }^{1}$ Department of Astronomy and Space Science, Kyung Hee University, Yongin 446-701, Korea \\ E-mail:mjang@khu.ac.kr \\ ${ }^{2}$ School of Space Research, Kyung Hee University, Yongin 446-701, Korea \\ E-mail:gchoe@khu.ac.kr \\ (Received December 02, 2009; Accepted December 27, 2009)
}

\begin{abstract}
A method of estimating the lower bound of coronal magnetic field strength in the neighborhood of an ejecting plasmoid is presented. Based on the assumption that the plasma ejecta is within a magnetic island, an analytical expression for the force acting on the ejecta is derived. The method is applied to a limb coronal mass ejection event, and a lower bound of the magnetic field strength just below the CME core is estimated. The method is expected to provide useful information on the strength of reconnecting magnetic field if applied to X-ray plasma ejecta.
\end{abstract}

Key words : methods: analytical — methods: data analysis — Sun: magnetic fields — Sun: coronal mass ejections — (magnetohydrodynamics:) MHD

\section{INTRODUCTION}

Variation of the space environment in the heliosphere, so-called space weather change, is caused by solar magnetic activities. Therefore, measuring solar magnetic fields is a primary task in the studies of solar activities and consequent space weather variations. The most gigantic and influential solar activities such as coronal mass ejections (CMEs) and solar flares take place in the solar corona, where magnetic energy is dominant over kinetic, thermal and gravitational energies of plasmas. However, measurement of solar magnetic fields is mostly made in the solar photosphere and chromosphere using spectropolarimetry (for review see e.g., Solanki 1993). Direct measurement of coronal magnetic fields is very difficult, if not totally impossible, with the current level of technology. There have been quite a few attempts to measure coronal magnetic fields, not for practical purposes, but rather for testing the proposed techniques. The strengths and weaknesses of most direct and indirect methods of coronal magnetic field measurement so far proposed have been reviewed in detail in Judge et al. (2001) and Cargill (2009). The most promising method for general magnetic field survey in the future would be spectropolarimetry of coronal emission lines (Lin et al. 2000, 2004, Tomczyk et al. 2008), but the current status is just testing it for some local regions of the corona.

The magnetic field near a reconnecting current sheet in a solar flare or around an ejecting plasma structure has its own interest and significance because it can be a touchstone of theories of magnetic reconnection and so-

Corresponding Author: G. S. Choe lar eruption and can also serve as a constraint or an input parameter of their theoretical and numerical models. The magnetic field strength in an active region can most effectively be measured by gyroresonance emission (Lee 2007), but this technique can be applied to a strong field region ( $>100 \mathrm{G}$ ) only, because the earth's atmosphere blocks frequencies generated in a weaker magnetic field. When a shock is present in a solar eruptive event, the split-band structure of type II bursts may be used to estimate the magnetic field strength along the path of the type II disturbance (Smerd et al. 1975, Cho et al. 2007). The amount of band splitting is a measure of the Alfvén Mach number. Therefore, if the plasma density is somehow given, we can obtain the field strength. However, the ambiguity in the location and the path of the type II generating shock limits the applicability of this method (Klein et al. 1999). Recently, Kim et al. (2008) have estimated the magnetic field strength in spicule regions from the MHD kink mode-like wave properties measured by Hinode SOT and an assumed spicule plasma density. Although this coronal seismology technique has a good prospect in field strength measurement, its use is limited to the regions showing detectable oscillations.

In this paper, we present a new method of estimating the lower limit of coronal magnetic field strength using the plasma ejecta motion during a solar eruption. This method is based on the theoretical observation that the Lorentz force acting on a magnetic island is a surface integral of magnetic pressure. The plasma ejecta appearing in solar eruption is believed to be embedded in a flux rope, which can be reasonably approximated by a magnetic island. With this method, we can estimate 
a lower bound of magnetic field strength just below or above the plasmoid depending on whether it is under acceleration or deceleration.

In Section II, our method of estimating the coronal field strength is expounded, and in Section III, the method is applied to a limb CME event observed by $\mathrm{SOHO} / \mathrm{LASCO} \mathrm{C} 2$ to compute a lower bound of the magnetic field strength below a plasma ejecta. Finally, a brief summary and discussion is given in Section IV.

\section{A METHOD OF ESTMATING CORO- NAL MAGNETIC FIELD STRENGTH}

It is generally believed that a magnetic flux rope is a main constituent of a solar eruptive event. It has long been observed that an erupting prominence often reveals a helical structure in $\mathrm{H}_{\alpha}$, which is regarded to delineate magnetic field lines. It is also generally believed that the magnetic field in the central part of a CME has a flux rope structure (Hundhausen 1999, Chen et al. 2000). In X-ray observations of solar flares, a plasma blob, so-called plasmoid, is sometimes ejected, which is also considered to reside in a flux rope (Ohyama \& Shibata 1998, Kim et al. 2005). In the present-day standard model of eruptive flares, so-called CSHKP model (Sturrock 1992), a rising flux rope is surrounded by line-tied fields and these fields reconnect in the current sheet underneath the flux rope. The magnetic reconnection accelerates the flux rope and the flux rope rising facilitates the magnetic reconnection. Thus, these two have a feedback effect (Shibata et al. 1995, Choe \& Cheng 2000). To understand the magnetic reconnection process and the plasmoid motion quantitatively, the information on the magnetic field strength is indispensable.

The field configuration in a typical solar eruptive event is sketched in Figure 1a. If we observe a limb event, we are likely to see the apex part of the flux rope. Let us consider a volume like a slab or a bent cylinder in the middle of the flux rope. The girth of the slab $\left(S_{3}\right)$ is set to be a flux surface such that magnetic field vectors are tangential to the surface. It should be noted that $S_{3}$ need not be the outer boundary of the flux rope, but can be any flux surface within the flux rope. Assume that the flux surfaces of the flux rope have a left-right symmetry. Surfaces $S_{1}$ and $S_{2}$ are assumed to be parallel and equally displaced from the central cross-section of the flux rope. We employ a Cartesian coordinate system, in which $\hat{\boldsymbol{z}}$ is directed from the center of the Sun to the flux rope apex, $\hat{\boldsymbol{y}}$ is the tangent vector to the flux rope magnetic axis at its apex directed from the left to the right, and $\hat{\boldsymbol{x}}$ is the normal vector to the plane spanned by the magnetic axis of the flux rope such that $\hat{\boldsymbol{x}}=\hat{\boldsymbol{y}} \times \hat{\boldsymbol{z}}$. Thus, $\hat{\boldsymbol{n}_{1}}=\hat{\boldsymbol{y}}$, and $\hat{\boldsymbol{n}_{2}}=-\hat{\boldsymbol{y}}$. Then, the force acting on the slab is given by (Choe \& Lee 1996)

$$
\boldsymbol{F}=\boldsymbol{F}_{L P}+\boldsymbol{F}_{G}
$$

$$
=\int_{V} \nabla \cdot \mathbf{T} d V-\int \rho g \hat{\boldsymbol{z}} d V
$$

where $\boldsymbol{F}_{L P}$ is the Lorentz force plus the pressure gradient force, $\boldsymbol{F}_{G}$ is the gravitational force, $\rho$ is the plasma density, $g$ is the gravitational acceleration, and $\mathbf{T}$ is the Maxwell stress tensor minus the isotropic plasma pressure tensor such that

$$
\mathbf{T}=\boldsymbol{B} \boldsymbol{B}-\left(\frac{B^{2}}{8 \pi}+P\right) \mathbf{I}
$$

In this paper, the Gaussian (cgs) unit system is employed. Applying the divergence theorem, we have

$$
\boldsymbol{F}_{L P}=\oint \hat{\boldsymbol{n}} \cdot \boldsymbol{B} \boldsymbol{B} d S-\oint \hat{\boldsymbol{n}}\left(\frac{B^{2}}{8 \pi}+P\right) d S,
$$

where $\hat{\boldsymbol{n}}$ is the outward normal vector. Owing to the left-right symmetry of the slab and the parallelness of $S_{1}$ and $S_{2}$, the surface integrals over $S_{1}$ and $S_{2}$ cancel each other. In the remaining surface integral over $S_{3}$, the first term vanishes since $S_{3}$ is a flux surface in which $\hat{\boldsymbol{n}} \cdot \boldsymbol{B}=0$. This implies that the magnetic tension does not contribute any force on this slab. Now we assume that the slab is thin enough for us to ignore the curvature of the magnetic axis within the slab. This assumption is valid as long as the depth of the plasma emitting (or scattering) light toward us in a limb event is small compared to the radius of curvature of the flux rope magnetic axis. In this case, we can ignore the $y$ dependence of physical quantities within the slab. If we denote the thickness of the slab by $D$, the force acting on the unit $y$-length of the slab is $\boldsymbol{f}=\boldsymbol{F} / D$. Now the surface integral over $S_{3}$ is $D$ times the line integral along the projection of $S_{3}$ onto the $x z$-plane. If we take the line element vector $d \boldsymbol{l}=d l \hat{\boldsymbol{l}}$ such that $\hat{\boldsymbol{l}}=\hat{\boldsymbol{y}} \times \hat{\boldsymbol{n}}$, then

$$
\hat{\boldsymbol{z}} \cdot \hat{\boldsymbol{n}}=\hat{\boldsymbol{z}} \cdot \hat{\boldsymbol{l}} \times \hat{\boldsymbol{y}}=\hat{\boldsymbol{x}} \cdot \hat{\boldsymbol{l}}=\frac{d x}{d l} .
$$

Thus, the $z$-component of the force acting on the slab with unit $y$-depth is

$$
\begin{aligned}
f_{z}= & \hat{\boldsymbol{z}} \cdot \boldsymbol{F} / D \\
= & -\oint\left(\frac{B^{2}}{8 \pi}+P\right) d x+\hat{\boldsymbol{z}} \cdot \boldsymbol{F}_{G} / D \\
= & {\left[\int_{x_{\min }}^{x_{\max }}\left(\frac{B^{2}}{8 \pi}+P\right) d x\right]_{l} } \\
& -\left[\int_{x_{\min }}^{x_{\max }}\left(\frac{B^{2}}{8 \pi}+P\right) d x\right]_{u} \\
& -\int_{S} \rho g d S,
\end{aligned}
$$

where the subscript $l$ denotes the lower part of the slab, the subscript $u$ denotes the upper part of the slab, $x_{\text {min }}$ is the lower bound of the $x$-coordinate of the slab, $x_{\max }$ is the upper bound of the $x$-coordinate of the slab, and 
(a)

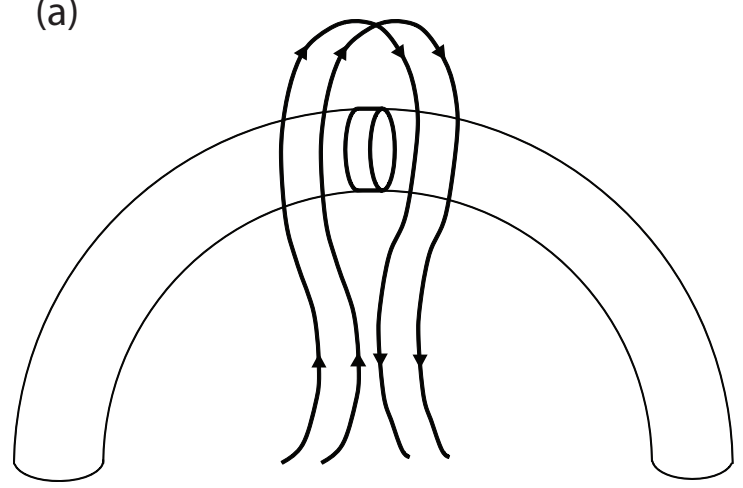

(b)

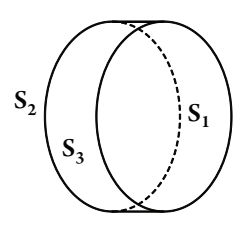

(c)

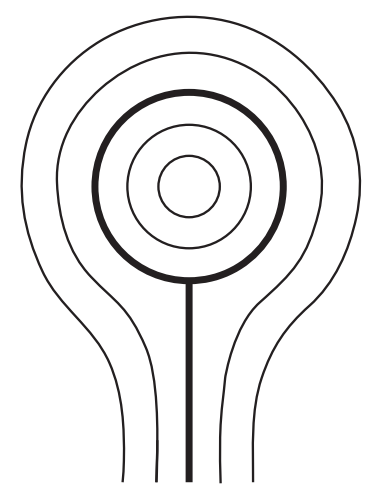

Fig. 1. - Sketch of field configuration in solar eruption. (a) A flux rope surrounded by line-tied fields. (b) A cylinder-like volume near the flux rope apex. (c) The cross-section of the whole configuration with a magnetic island and surrounding fields.

in the last integral for gravity, $S=S_{1}=S_{2}$. It is to be noted that this equation holds even if the surface $S_{3}$ is a singular current sheet, because the total pressure is continuous across a current sheet.

Equation (5) is an exact expression when viscosity and other body forces are ignorable. We, however, have too few pieces of information to make use of it for estimating magnetic field strength. For practical purposes, we define the following quantities:

$$
\begin{gathered}
\Delta x=x_{\max }-x_{\min }, \\
<P_{B, l / u}>=\frac{1}{\Delta x}\left[\int_{x_{\min }}^{x_{\max }} \frac{B^{2}}{8 \pi} d x\right]_{l / u}, \\
<\Delta P_{B}>=<P_{B, l}>-<P_{B, u}>, \\
<P_{P, l / u}>=\frac{1}{\Delta x}\left[\int_{x_{\min }}^{x_{\max }} P d x\right]_{l / u}, \\
<\Delta P_{P}>=<P_{P, l}>-<P_{P, u}>.
\end{gathered}
$$

We also define the mass per unit $y$-depth $m=\int_{S} \rho d S$, the acceleration of the center of mass $\boldsymbol{a}=\boldsymbol{f} / \mathrm{m}$, and the mean gravity $\langle g\rangle=(1 / m) \int \rho g d S$, which is the gravitational acceleration of the center of mass. Then, equation (5) is

$$
<\Delta P_{B}>+<\Delta P_{P}>=\frac{m}{\Delta x}\left(a_{z}+<g>\right) .
$$

Although looking simple, this equation is also exact. Generally, the quantities on the right-hand side can be obtained from observations. The left-hand side is actually made of four quantities: $\left\langle P_{B, l}\right\rangle,\left\langle P_{B, u}\right\rangle$, $\left\langle P_{P, l}\right\rangle$, and $\left\langle P_{P, u}\right\rangle$. If we can somehow identify each of these quantities, a sort of mean magnetic field strength below or above the plasmoid is

$$
\begin{aligned}
<B_{l / u}> & =\left\{8 \pi \left[<P_{B, u / l}>\right.\right. \\
\pm & \left((m / \Delta x)\left(a_{z}+<g>\right)\right. \\
& \left.\left.\left.-<\Delta P_{P}>\right)\right]\right\}^{\frac{1}{2}}
\end{aligned}
$$

where the right-hand side quantity is the larger one between $\left\langle B_{l}>\right.$ and $<B_{u}>$.

In general, we are not given enough information to isolate $\left\langle P_{B, l}>,\left\langle P_{B, u}>\right.\right.$, and $\left\langle\Delta P_{P}\right\rangle$. In many cases, $\left\langle\Delta P_{P}\right\rangle$ is difficult to estimate, and we cannot but make some rough assumptions about the plasma pressure. One such assumption would be that $<\Delta P_{P}>$ be ignorable. This is a legitimate case in a low $\beta$ plasma in the low corona. In a very low $\beta$ plasma, it is also likely that the gravity term is smaller than the magnetic pressure term. Another possibility might be that the gravity and the plasma pressure be more or less in equilibrium in the vertical direction so that

$$
m<g>/ \Delta x-<\Delta P_{P}>\approx 0 .
$$

Under this assumption, equation (12) becomes

$$
<B_{l / u}>=\left\{8 \pi\left[\left\langle P_{B, u / l}\right\rangle \pm\left(m a_{z} / \Delta x\right)\right]\right\}^{\frac{1}{2}} .
$$

No matter what assumption is made, what we can measure is the magnetic pressure difference between below and above the plasmoid. With the above formulation only, estimating the magnetic pressure (field strength squared) on both sides is impossible. Accepting this limitation, we may have to be contented with finding the lower bound of magnetic field strength in order to be on a safe side. In other words,

$$
<B_{l / u}>z\left(8 \pi m\left|a_{z}\right| / \Delta x\right)^{1 / 2}
$$

Since this lower bound is obtained by setting the smaller field strength to zero, it may be too low to be a practical estimate. In an effort to compensate for this deficit, we may take a maximum absolute value of acceleration out of a short sequence (maybe three of four) of measured values, roughly assuming that the field strength on the larger side does not change much during this sequence. In the following section, we demonstrate how to use this procedure to estimate the magnetic field strength below a real plasmoid. 


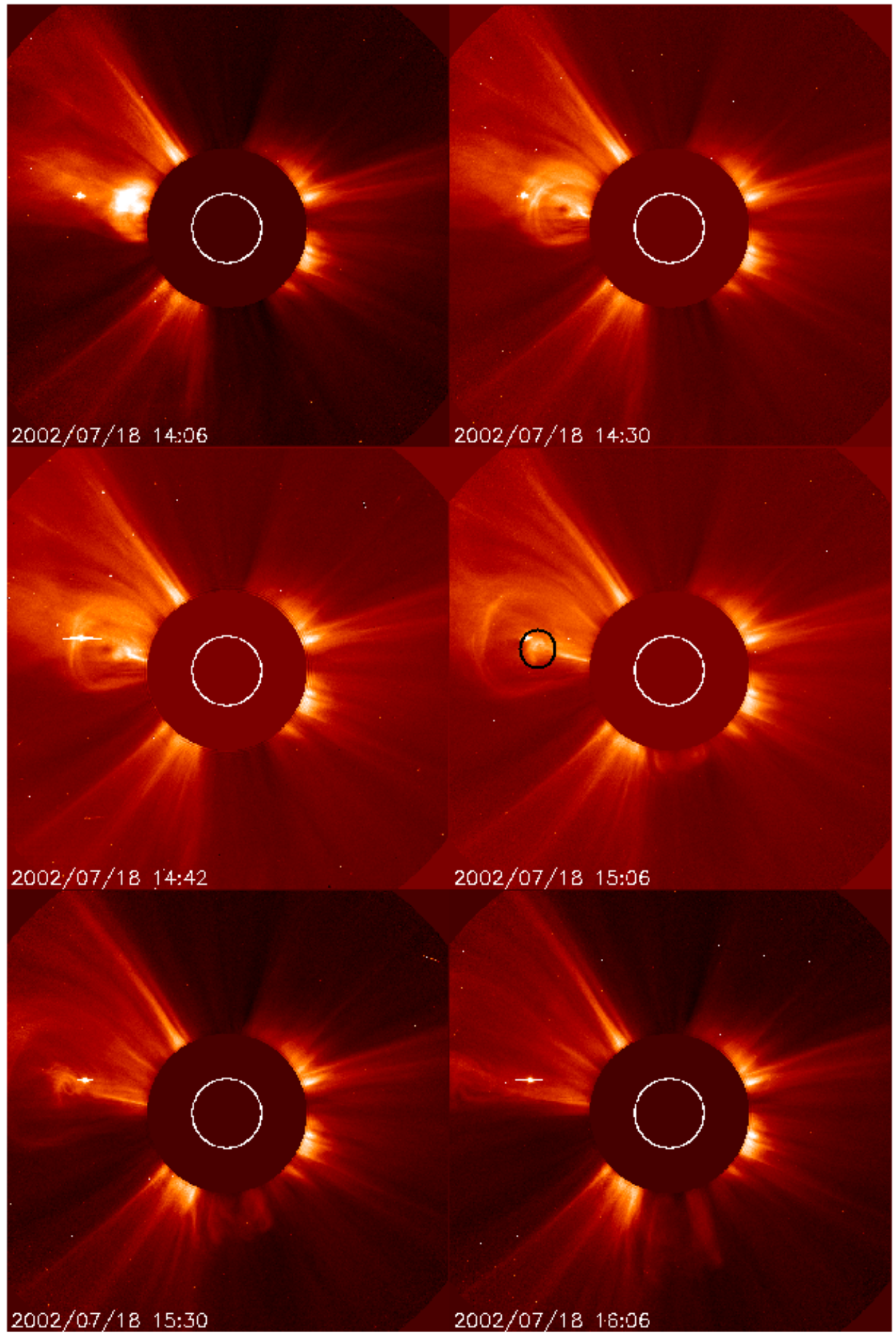

Fig. 2.- LASCO C2 images of a CME on 2002 July 18. The area encircled in black contains a CME core and a cavity and is likely to be within a flux rope. 


\section{APPLICATION TO A LIMB CME ON 2002 JULY 18}

To apply the aforementioned method to estimation of magnetic field strength, one needs to know the acceleration of an X-ray plasmoid or an island-like CME structure. Therefore, it is more convenient to deal with a limb event than a disk-center event or a halo event. In the latter case, we need a directional correction of the velocity projected on the sky plane. In the SOHO/LASCO CME catalogue (Yashiro et al. 2004, http://cdaw.gsfc.nasa.gov/CME_list/), the CME that first appeared in the LASCO C2 field of view at 2002 July 18 14:06:08 is selected for our purpose. It is a limb event with a quasi-two-dimensional configuration resembling Figure 1c as in the standard model of eruptive flares. It shows an island-like structure with a density enhanced lower part and a cavity-like upper part, a steady clear current sheet structure below it and a CME frontal loop surrounding all these (Fig. 2).

\section{(a) Measurement of Acceleration}

Table 1 lists the basic observational quantities of the event. Here, the height is measured from the solar center to the center of the core part. Since the densityenhanced part and the upper cavity are reasonably believed to reside in a flux rope, we take the area encircled in black in the fourth image of Figure 2 as the projection of the volume in question. The acceleration can most simply be determined by a three-point quadratic function fitting. The acceleration values obtained with this method are listed in Table 1 . In this paper, we are particularly interested in the maximum and minimum (more exactly, negative maximum) values of acceleration because the positive maximum value can give us a lower bound of field strength as large as we can have below the plasmoid and the negative maximum value can give us a lower bound of field strength as large as we can have above the plasmoid. In Table 1 the maximum value of acceleration is $6.17 \times 10^{3} \mathrm{~cm} \mathrm{~s}^{-2}$ at 14:42:05, when the center of our interested volume is at a distance of $3.06 R_{\odot}$ from the solar center. The minimum value is uncertain because the plasmoid starts to decelerate before 15:06:08 and keeps decelerating. At 15:06:08, the acceleration is $-2.07 \times 10^{4} \mathrm{~cm} \mathrm{~s}^{-2}$ and the height of the volume center is $4.21 R_{\odot}$. The maximum value obtained with a three-point quadratic fitting method is always less than the real maximum value in the same time interval, and the minimum value obtained with the same method is always greater than the real minimum value. To estimate the better value of the maximum acceleration, we have also used a fourth-order polynomial fitting method, and the maximum value thus obtained is $1.01 \times 10^{4} \mathrm{~cm} \mathrm{~s}^{-2}$ at 14:39:06, when the height is $2.83 R_{\odot}$ (see Fig. 3 ).

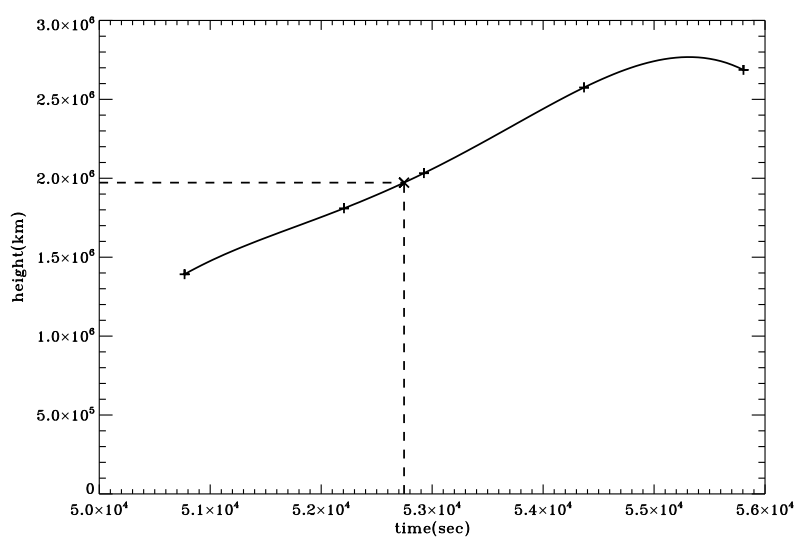

Fig. 3. - The height of the CME core interpolated by a fourth order polynomial fitting. The maximum acceleration occurs at 14:39:06.

\section{(b) Estimation of Mass Per Unit Depth}

Compared to the measurement of acceleration, the estimation of the CME mass per unit depth is not so straightforward. In general, CME masses are estimated using data from white-light coronagraphs (Poland et al. 1981, Howard et al. 1985). Coronagraphs observe the integral of the photospheric light that is Thomsonscattered by the CME electrons. Thus, the observed brightness at each area element in the sky plane gives information on the total number of electrons along the line of sight (LOS). In this paper, we have derived the mass of the CME using the method described in Vourlidas et al. (2000). Since a white light CME is defined as brightness increase relative to the background, we use pre-event-subtracted images to remove the background corona. Before the CME of our interest took place, there had been another CME, which was first observed by LASCO C2 at 12:06:09. This situation compels us to use the LASCO C2 image at 11:42:05 as the pre-event image. From the brightness excess thus obtained and the Thomson scattering function by Billings (1966), we have calculated the total brightness and the polarized brightness. The ratio of the total brightness to the polarized brightness gives the total number of electrons along the LOS. Assuming that the CME plasma composition is $10 \%$ helium and $90 \%$ hydrogen and $1.97 \times 10^{-24} \mathrm{~g}$ of mass is thus assigned to each electron, we obtain the total mass of the CME. To calculate the mass contained in the interested volume, we have summed up the masses of the pixels encompassing the volume. Unfortunately, there was a planet seen in the volume. To avoid this planet, we have selected the area excluding the planet using the ROI (Region of Interest) routine in IDL. The mass of each pixel or the excess electron column density (in $\mathrm{cm}^{-2}$ ) can be converted to electron volume density (in $\mathrm{cm}^{-3}$ ) when the depth of the structure along the LOS is known. We have simply assumed that the depth along 
TABLE 1.

Observed and Derived Physical Quantities of the CME on 2002 July 18

\begin{tabular}{cccccccc}
\hline \hline $\begin{array}{c}\text { TIME } \\
(\mathrm{UT})\end{array}$ & $\begin{array}{c}\text { HEIGHT } \\
\left(R_{\odot}\right)\end{array}$ & $\begin{array}{c}\text { VELOCITY } \\
\left(\times 10^{7} \mathrm{~cm} \mathrm{~s}^{-1}\right)\end{array}$ & $\begin{array}{c}\text { ACCELERATION } \\
\left(\times 10^{3} \mathrm{~cm} \mathrm{~s}^{-2}\right)\end{array}$ & $\begin{array}{c}\text { SIZE } \\
\left(\times 10^{10} \mathrm{~cm}\right)\end{array}$ & $\begin{array}{c}\text { MASS } \\
\left(\times 10^{13} \mathrm{~g}\right)\end{array}$ & $\begin{array}{c}N_{e} \\
\left(\times 10^{5} \mathrm{~cm}^{-3}\right)\end{array}$ & $\begin{array}{c}m=M / D \\
\left(\times 10^{3} \mathrm{~g} \mathrm{~cm}^{-1}\right)\end{array}$ \\
\hline $14: 30: 05$ & 2.80 & 2.93 & 1.53 & 3.73 & 6.92 & 7.71 & 1.85 \\
$14: 42: 05$ & 3.06 & 3.09 & 6.17 & 3.47 & 6.27 & 10.07 & 1.81 \\
$15: 06: 08$ & 4.21 & 3.76 & -20.7 & 6.25 & 16.26 & 4.40 & 2.60 \\
$15: 30: 05$ & 4.83 & 0.78 & - & 8.68 & 18.31 & 2.71 & 2.11 \\
\hline
\end{tabular}

${ }^{a}$ The mean velocity between the previous observing time and the given time

the LOS is the same as the angular latitudinal span. With this rough assumption, we have computed the electron number density and the total mass per unit depth along the LOS (in $\mathrm{g} \mathrm{cm}^{-1}$ ). The assumption we make on the uncertain depth may possibly underestimate the actual mass (Vourlidas et al. 2000, Lugaz et al. 2005). An underestimate of the CME mass would consequently lead to an underestimate of field strength. As long as we seek a lower bound of field strength, an underestimate does not spoil the validity of our conclusion. The values of the total mass per unit depth obtained by the above procedure are given in Table 1 . The value at the first appearance of the CME (14:06:08) is exceptionally large because the CME frontal loop and the core are not well resolved at this time. Thus, we do not consider this value. The later values are located between $1.73-1.85 \times 10^{3} \mathrm{~g} \mathrm{~cm}^{-1}$ except the value at 15:06:08 $\left(2.61 \times 10^{3} \mathrm{~g} \mathrm{~cm}^{-1}\right)$, at which the presence of a planet within the area of interest makes our mass estimate unreliable.

\section{(c) A Lower Bound of Magnetic Field Strength}

A lower bound of magnetic field strength below the plasmoid is obtained from equation (15) with the maximum acceleration value, the mass per unit depth of the plasmoid computed above, and the width of the plasmoid. For this calculation, we first use the values at 14:42:05 obtained from the three-point quadratic fitting, i.e., $a_{z}=6.17 \times 10^{3} \mathrm{~cm} \mathrm{~s}^{-1}, m=1.81 \times 10^{3} \mathrm{~g} \mathrm{~cm}^{-1}$, and $\Delta x=3.47 \times 10^{10} \mathrm{~cm}$. This computation yields a lower bound of magnetic field strength below the plasmoid; $\left\langle B_{l}>\gtrsim 0.0899 \mathrm{G}\right.$. If we use the maximum acceleration obtained with the fourth order polynomial interpolation $\left(a_{z}=1.01 \times 10^{4} \mathrm{~cm} \mathrm{~s}^{-2}\right.$ at 14:39:06) and the mass and the width obtained with the data at 14:42:05, we have $\left\langle B_{l}\right\rangle \gtrsim 0.115 \mathrm{G}$. In order for our use of equation (15) to be justified, either the quasihydrostatic equilibrium assumption (eq. [13]) should be valid, or the gravitational acceleration and the plasma pressure, respectively, are small compared to the plasmoid acceleration and the magnetic pressure. The solar gravitational acceleration at the radius of $3.06 R_{\odot}$ is $2.93 \times 10^{3} \mathrm{~cm} \mathrm{~s}^{-2}$, which is about half the plasmoid acceleration of $6.17 \times 10^{3} \mathrm{~cm} \mathrm{~s}^{-1}$. The gravitational acceleration, however, increases the field strength esti- mate below the plasmoid unless the plasma pressure is dominant over the magnetic pressure. To evaluate the plasma pressure, we need to know the number of particles per electron. This value is less than 2 because there are multiply ionized heavy elements, but using the value 2 is fine if we want an upper bound. For temperature, we take $T=1.5 \times 10^{6} \mathrm{~K}$. In Table 1 , the largest value of the electron density is $1.01 \times 10^{6} \mathrm{~cm}^{-3}$. With these values, the maximum plasma pressure is evaluated to be $4.19 \times 10^{-4} \mathrm{dyn}_{\mathrm{cm}}^{-2}$, which is slightly smaller than the lower bound of magnetic pressure $(0.115 \mathrm{G})^{2} /(8 \pi)=5.26 \times 10^{-4} \mathrm{dyn}_{\mathrm{cm}}^{-2}$. Therefore, the use of equation (15) can be more or less justified in this case. It is anyhow regrettable that no other measurement of coronal field strength at such a high altitude has yet been reported and there is no reference value to be compared with our estimate.

\section{SUMMARY AND DISCUSSION}

We have presented a new method of estimating a lower bound of magnetic field strength near (in most cases below) a plasmoid in solar eruption. The method is based on the theoretical observation that the vertical force acting on a cylinder-like volume, whose lateral surface is a flux surface, is just the difference in total pressure (magnetic pressure plus plasma pressure) below and above the volume. We have applied this method to a limb CME that occurred on 2002 July 18 , and obtained a field strength lower bound of $\sim 0.1 \mathrm{G}$ below the CME core.

As mentioned earlier, equation (11) implies that only a total pressure difference exerts a force to a flux rope, but a magnetic tension does not. This may sound counter-intuitive because a magnetic tension is considered to be a major force to expel reconnection outflows in which a plasmoid may reside. This conventional view is correct if the volume of our interest has an open flux in some part of its boundary as usually depicted in a reconnection picture with an X-shaped separatrix (e.g., Petschek 1964). As for a volume, whose boundary is a flux surface, the view is incorrect. The magnetic tension over the whole boundary cancels out. Of course, it may act to shrink the volume if the guide field or the plasma pressure inside the volume is not strong enough, but it cannot accelerate the center of mass of the vol- 
ume in any case. In our case, only part of the boundary is a flux surface $\left(S_{3}\right.$ in Fig. 1b), but the the symmetry of the volume makes the forces on $S_{1}$ and $S_{2}$ cancel out. If we cannot ignore the curvature of the volume or if a self-closed magnetic structure, for example, a magnetic torus, were to exist in the solar atmosphere, we would have a more general relation similar to Equation (11) as follows.

$$
<\Delta P_{B}>+<\Delta P_{P}>=\frac{M}{\Delta A}\left(a_{z}+<g>\right),
$$

where $M$ is the mass in the volume, $\Delta A$ is the projected area of the volume onto the $x y$-plane,

$$
<\Delta P_{B}>=\frac{1}{\Delta A}\left[\int_{S_{l}} \frac{B^{2}}{8 \pi} d x d y-\int_{S_{u}} \frac{B^{2}}{8 \pi} d x d y\right]
$$

and

$$
<\Delta P_{P}>=\frac{1}{\Delta A}\left[\int_{S_{l}} P d x d y-\int_{S_{u}} P d x d y\right],
$$

in which $S_{l}$ is the part of the boundary surface facing downward $(\hat{\boldsymbol{z}} \cdot \hat{\boldsymbol{n}}<0)$ and $S_{u}$ the part facing upward $(\hat{\boldsymbol{z}} \cdot \hat{\boldsymbol{n}}>0)$.

If we apply our proposed procedure to the deceleration phase of the same CME with $a_{z}=-2.07 \times$ $10^{4} \mathrm{~cm} \mathrm{~s}^{-2}$ at 15:06:08, $m=1.81 \times 10^{3} \mathrm{~g} \mathrm{~cm}^{-1}$ (instead of the doubtful value $2.6 \times 10^{3} \mathrm{~g} \mathrm{~cm}^{-1}$ ), and $\Delta x=6.25 \times 10^{10} \mathrm{~cm}$, we have a lower bound of the magnetic field strength above the plasmoid volume including the cavity; $\left\langle B_{u}\right\rangle \gtrsim 0.123 \mathrm{G}$. However, the LASCO image at 15:06:08 does not seem to show any remarkable compression above the plasmoid. If there is indeed no compression above the volume, the deceleration must come from another force. The most plausible candidate of this force would be an aerodynamic drag. Although the Reynolds number of the coronal plasma is very large, there can be a boundary layer, within which viscous force is not negligible (e.g., Batchelor 1967). Since the aerodynamic drag is always opposite to the plasmoid motion, the lower bound of the field strength above the rising plasmoid may well be lower than the estimated value. This is the reason why we take a reserved position in applying our method to the upstream side in the reference frame of the moving plasmoid.

Although we have demonstrated the use of our method with a LASCO CME, the plasma $\beta$ near unity in the outer corona does not allow us strong confidence in the estimated value. Also, the low time cadence of the coronagraph observation does not enable us to make a more accurate measurement of acceleration. The method would better suit estimation of magnetic field below an X-ray plasmoid because it is observed in the low $\beta$ corona. It is also an advantage that $\mathrm{X}$-ray observations have a far higher time cadence. Moreover, an X-ray plasmoid is smaller and located in the lower corona than the coronagraph-observed structure; the viscous drag is therefore negligible. The strength of the reconnecting magnetic field below an X-ray plasmoid is a highly sought quantity in the current solar research and can be estimated with our method. Applying the method to other types of observations than those by coronagraphs will be pursued in the future.

\section{ACKNOWLEDGEMENTS}

The authors thank the anonymous referee for constructive comments and suggestions, which helped to improve the manuscript. This work has been supported by the WCU grant (No. R31-10016) funded by the Korean Ministry of Education, Science and Technology. G. S. C. was also supported by the Korea Research Foundation grant funded by the Korean Government (KRF-2007-313-C00324). K.-S. K. expresses his thanks to Kyung Hee University for granting him a sabbatical, during which his part of this research was performed. He also extends his sincere gratitude to Profs. K. Shibata, K. Ichimoto and R. Kitai, and other members of Kwasan and Hida Observatories of Kyoto University for the generous support and hospitality provided to him during his stay there.

\section{REFERENCES}

Batchelor, G. K., 1967, An Introduction to Fluid Dynamics (Cambridge: Cambridge University Press)

Billings, D. E., 1966, A Guide to the Solar Corona (New York: Academic Press)

Cargill, P. J., 2009, Coronal Magnetism: Difficulties and Prospects, Space Sci. Rev., 144, 413

Chen, J., et al., 2000, Magnetic Geometry and Dynamics of the Fast Coronal Mass Ejection of 1997 September 9, ApJ, 533, 481

Cho, K.-S., Lee, J., Gary, D. E., Moon, Y.-J., \& Park, Y. D. 2007, Magnetic Field Strength in the Solar Corona from Type II Band Splitting, ApJ, 665, 799

Choe, G. S. \& Cheng, C. Z., 2000, A Model of Solar Flares and Their Homologous Behavior, ApJ, 541, 449

Choe, G. S. \& Lee, L. C., 1996, Evolution of Solar Magnetic Arcades. II. Effect of Resistivity and Solar Eruptive Processes, ApJ, 472, 372

Howard, R. A., Sheeley, N. R., Jr., Michels, D. J., \& Koomen, M. J., 1985, Coronal Mass Ejections 1979-1981, J. Geophys. Res., 90, 8173

Hundhausen, A. J., 1999, Coronal Mass Ejections, in The Many Faces of the Sun: A Summary of the Results from NASA's Solar Maximum Mission, ed. K. T. Strong, J. L. R. Saba, B. M. Haisch, \& J. T. Schmelz (New York: Springer-Verlag), 143

Judge, P. G., Casini, R., Tomczyk, S., Edwards, D. P., \& Francis, E., 2001, Coronal Magnetometry: A Feasibility Study, NCAR/TN-446+STR (Boulder: National Center for Atmospheric Research) 
Kim, Y.-H., Bong, S.-C., Park, Y. D., Cho, K.-S., Moon, Y.-J., \& Suematsu, Y., 2008, Estimation of Spicule Magnetic Field Using Observed MHD Waves by the Hinode SOT, JKAS, 41, 173

Kim, Y.-H., Moon, Y.-J., Cho, K.-S., Kim, K.-S., \& Park, Y. D., 2005, A Study of Flare-associated XRay Plasma Ejections. I. Association with Coronal Mass Ejections, ApJ, 622, 1240

Klein, K.-L., Khan, J. I., Vilmer, N., Delouis, J.-M., \& Aurass, H., 1999, X-ray and Radio Evidence on the Origin of a Coronal Shock Wave, A\&A, 346, L53

Lee, J., 2007, Radio Emissions from Solar Active Regions, Space Sci. Rev., 133, 73

Lin, H., Kuhn, J. R., \& Coulter, R., 2004, Coronal Magnetic Field Measurements, ApJ, 613, L177

Lin, H., Penn, M. J., \& Tomczyk, S., 2000, A New Precise Measurement of the Coronal Magnetic Field Strength, ApJ, 541, L83

Lugaz, N., Manchester, W. B., IV, \& Gombosi, T. I., 2005, The Evolution of Coronal Mass Ejection Density Structures, ApJ, 627, 1019

Ohyama, M., \& Shibata, K. 1998, X-Ray Plasma Ejection Associated with an Impulsive Flare on 1992 October 5: Physical Conditions of X-Ray Plasma Ejection, ApJ, 499, 934

Petschek, H. E., 1964, Magnetic Field Annihilation, in Proceedings of the AAS-NASA Symposium on the Physics of Solar Flares, NASA Special Publication 50, ed. W. N. Hess (Washington, DC: NASA), 425

Poland, A. I., Howard, R. A., Koomen, M. J., Michels, D. J., \& Sheeley, N. R., Jr., 1981, Coronal Transients near Sunspot Maximum, Sol. Phys., 69, 169

Shibata, K., Masuda, S., Hara, H., Yokoyama, T., Tsuneta, S., Kosugi, T., \& Ogwara, Y., 1995, HotPlasma Ejections Associated with Compact-Loop Solar Flares, ApJ, 451, L83

Smerd, S. F., Sheridan, K. V., \& Stewart, R. T., 1975, Split-Band Structure in Type II Radio Bursts from the Sun, Astrophys. Lett., 16, 23

Solanki, S. K., 1993, Smallscale Solar Magnetic Fields - an Overview, Space Sci. Rev., 63, 1

Sturrock, P. A., 1992, The Emerging Picture of Eruptive Solar Flares, in Eruptive Solar Flares, IAU Colloquium 133, Lecture Notes in Physics, 399, ed. Z. Švestka, B. V. Jackson, \& M. E. Machado (Berlin: Springer-Verlag), 397

Tomczyk, S., et al., 2008, An Instrument to Measure Coronal Emission Line Polarization, Sol. Phys., 247, 411

Vourlidas, A., Subramanian, P., Dere, K. P., \& Howard, R. A., 2000, Large-Angle Spectrometric Coronagraph Measurements of the Energetics of Coronal Mass Ejections, ApJ, 534, 456
Yashiro, S., Gopalswamy, N., Michalek, G., St. Cyr, O. C., Plunkett, S. P., Rich, N. B., \& Howard, R. A., 2004, A Catalog of White Light Coronal Mass Ejections Observed by the SOHO Spacecraft, J. Geophys. Res., 109, 7105 